\title{
Chromium(III) and iron(III) inhibits replication of DNA and RNA viruses
}

\author{
Sylwia Terpiłowska $\cdot$ Andrzej Krzysztof Siwicki
}

Received: 27 February 2017 / Accepted: 6 June 2017 / Published online: 13 June 2017

(C) The Author(s) 2017. This article is an open access publication

\begin{abstract}
The aim of this study was to examine the effect of treating of chromium(III) and iron(III) and their combinations on Herpes Simplex Virus type 1 (HSV-1) and Bovine Viral Diarrhoea virus (BVDV) replication. The antiviral efficacies of chromium(III) and iron(III) on HSV-1 and BVDV were evaluated using Real Time PCR method. Moreover, the cytotoxicity of these microelements was examined using the MTT reduction assay. $\mathrm{The} \mathrm{IC}_{50}(50 \%$ inhibiotory concentration) for the chromium chloride was $1100 \mu \mathrm{M}$ for Hep- 2 cells and $1400 \mu \mathrm{M}$ for BT cells. The $\mathrm{IC}_{50}$ for the iron chloride was $1200 \mu \mathrm{M}$ for Hep-2 cells and more than $1400 \mu \mathrm{M}$ for BT cells. The concentration-dependent antiviral activity of chromium chloride and iron chloride against HSV-1 and BVDV viruses was observed. In cultures simultaneously treated with (1) $200 \mu \mathrm{M}$ of $\mathrm{CrCl}_{3}$ and $1000 \mu \mathrm{M}$ of $\mathrm{FeCl}_{3}$, (2) $1000 \mu \mathrm{M}$ of $\mathrm{CrCl}_{3}$ and $200 \mu \mathrm{M}$ of $\mathrm{FeCl}_{3}$, (3) $400 \mu \mathrm{M}$ of $\mathrm{CrCl}_{3}$ and $800 \mu \mathrm{M}$ of $\mathrm{FeCl}_{3}$, (4)
\end{abstract}

S. Terpiłowska $(\bowtie)$

Laboratory of Environmental Biology, Institute of Environmental Engineering, The John Paul II Catholic University of Lublin, Racławickie 14 Av, 20-950 Lublin, Poland

e-mail: sylter@kul.pl

A. K. Siwicki

Department of Microbiology and Clinical Immunology,

University of Warmia and Mazury in Olsztyn,

Oczapowskiego 13, Str., 10-957 Olsztyn, Poland

e-mail: siwicki@uwm.edu.pl
$800 \mu \mathrm{M}$ of $\mathrm{CrCl}_{3}$ and $400 \mu \mathrm{M}$ of $\mathrm{FeCl}_{3}$ a decrease in number of DNA or RNA copies was observed compared with control cells and cells incubated with chromium(III) and iron(III) used separately. The synergistic antiviral effects were observed for chromium(III) and iron(III) against HSV-1 and BVDV.

Keywords Chromium(III) - Iron(III) - BVDV · HSV-1

\section{Introduction}

The herpesviruses comprise a very large group of animal viruses, each one of which is usually specialized in nature to infect a particular species of mammal, marsupial, fish, bird, reptile, amphibian or even bivalve. All the studied herpesviruses share the three following characteristics: (1) typical morphology, (2) possession of a large genome consisting of single molecule of double stranded DNA ranging in size between 120 and $259 \mathrm{kbp}$, (3) the ability to follow productive infection to produce disease, as well as enter a latent phase in some cells of the infected natural host. This latent phase ensures survival of the viral genome throughout the lifetime of the particular infected individual and the ability to reenter the productive phase from time to time (Subak-Sharpe and Darga 1998).

The Herpes Simplex Virus type 1 (HSV-1) is a widely prevalent DNA virus that causes a range of 
human diseases. The most potent and well-studied anti HSV-1 agent is Acyclovir (ACV). However, ACV's anti-HSV ability is attenuated when initial treatment is delayed. Furthermore, in immunocompromised individuals, ACV-resistant HSV-1 strains often arise (He and Tam 2010).

The Bovine Viral Diarrhoea Virus (BVDV), a member of the genus Pestivirus in the Flaviviridae family, is associated with various diseases of cattle, including respiratory infections, gastrointestinal infections, and reproductive problems such as infertility, abortion, still birth, and weak calves. The BVDV has been reported in other animals, e.g. pigs, sheep, goats, deer, and in captive and free-living ruminants. The BVDVs are grouped into two genotypes: BVDV1 and BVDV2. The BVDV2 can be subdivided into two subgenotypes, whereas the BVDV in North America can be subdivided into two genotypes: BVDV1a and BVDV1b, though more than 11 subgenotypes were reported in Europe (Kim et al. 2009).

Symptoms of acute infection range from an inapparent to a severe course and can involve the respiratory, enteric, reproductive, immune and endocrine systems (Letellier and Kerkhofs 2003; Zhang et al. 2014). The infection can cause a decrease in milk production, a reduced reproductive performance and growth retardation (Chai et al. 2013). Infection in the early gestation period with BVDV may produce persistently infected calves that are responsible for the spread of virus in the herds (Kim et al. 2009).

The BVDV, has been a good model virus for investigating $\mathrm{HCV}$, which is a member of genus Hepacivirus, which belongs to the same family (Chai et al. 2013).

Therefore, the development of new anti-HSV and BVDV agents is important ( $\mathrm{He}$ and Tam 2010). Currently, there is no specific antiviral agent directed against BVDV (Chai et al. 2013).

Nutrition plays an important role in the development and also in the prevention of cancer, cardiovascular diseases, and diabetes. Chromium(III) and iron(III) are trace elements necessary for growth and normal functioning of cells. Moreover, it has been found that zinc inhibits rhinovirus $3 \mathrm{C}$ protease, consequently inhibiting viral replication in vitro (Haase et al. 2008). Moreover, another study has shown, that inorganic selenium species inhibits in vitro replication of Coxackie virus B5 strain (Cermelli et al. 2002). The aim of this study was to examine the effect of chromium(III) and iron(III) used separately and simultaneous treating of chromium(III) and iron(III) on HSV-1 an BVDV replication. The concentrations of chromium chloride and iron chloride for these studies were chosen on the basis of other reports (Mazzotti et al. 2001, 2002) and our earlier investigations (Terpiłowska and Siwicki 2009, 2010, 2012).

\section{Materials and methods}

\section{Chemicals}

The Eagle's Minimum Essential Medium (EMEM), the Dulbecco's Modified Eagle's Medium (DMEM), the heat-inactivated Fetal Bovine Serum (FBS) and the Horse Serum (HS) were purchased from The American Type Culture Collection (USA); Antibiotic/ antimycotic solution $(10,000 \mathrm{U} / \mathrm{ml}$ of penicillin, $10 \mathrm{mg} / \mathrm{ml}$ of streptomycin, $25 \mu \mathrm{g} / \mathrm{ml}$ of amphotericin B) and The MTT reduction assay-In vitro Toxicology Assay Kit MTT based (TOX-1) were purchased from Sigma Aldrich (Sigma Aldrich Inc., St. Louis, $\mathrm{MO}, \mathrm{USA})$; iron chloride $\left(\mathrm{FeCl}_{3} \times 6 \mathrm{H}_{2} \mathrm{O}\right)$ and chromium chloride $\left(\mathrm{CrCl}_{3} \times 6 \mathrm{H}_{2} 0\right)$ were purchased from Acros Organics (Belgium). Pathogenic Free DNA isolation Kit, Free RNA isolation Kit and Herpes Simplex Virus (HSV1/2) PCR Kit were purchased form Gene Proof (Czech Republic). Quantification of Bovine Viral Diarrhoea Virus Kit was purchased form PrimerDesign, Ltd. (United Kingdom).

Cells and virus strains

Human epithelial carcinoma HEp-2 cells (ATTC CCL-23) and Herpes Simplex Virus type1 (ATTC VR-1493) were purchased from the American Type Culture Collection (USA). Hep-2 cells were cultured in EMEM supplemented with 10\% (v/v) FBS, Antibiotic/antimycotic solution ( $1 \mathrm{ml}$ per $100 \mathrm{ml}$ of cell culture medium) at $37{ }^{\circ} \mathrm{C}$ in humidified atmosphere of $5 \% \mathrm{CO}_{2} / 95 \%$ air.

BT (turbinate) cells (ATTC CRL-1390) and Bovine Viral Diarrhea Virus (ATCC VR-534) were purchased from the American Type Culture Collection (USA). BT cells were cultured in DMEM supplemented with $10 \%$ (v/v) HS, Antibiotic/antimycotic solution (1 ml per $100 \mathrm{ml}$ of cell culture medium) at $37^{\circ} \mathrm{C}$ in humidified atmosphere of $5 \% \mathrm{CO}_{2} / 95 \%$ air. 
Cytotoxicity assay

Analyses of the in vitro cytotoxicity of chromium(III) and iron(III) were performed with an enzymatic assay (MTT assay) which is capable of quantifying the activity of mitochondrial enzymes in active and dividing cells. The MTT reduction assay is based on the enzymatic conversion of MTT (3-[4,5-dimethylthiazol-2-yl]-2,5-diphenyltetrazolium bromide) by the metabolically active cells in mitochondria. As a result, the MTT assay is a marker of mitochondrial function. The Hep- 2 or BT cells were cultured at $2 \times 10^{5}$ cells/ $\mathrm{ml}$ as adherent monolayers in plastic tissue-culture dishes in EMEM or DMEM, respectively, supplemented with $10 \%(\mathrm{v} / \mathrm{v})$ heat-inactivated FBS or HS, respectively, and Antibiotic/antimycotic solution ( $1 \mathrm{ml}$ per $100 \mathrm{ml}$ of cell culture medium). Cells were maintained at $37{ }^{\circ} \mathrm{C}$ in humidified incubator in atmosphere containing $5 \% \mathrm{CO}_{2}$. Cells were used for cytotoxicological assay during exponential phase of growth. After $24 \mathrm{~h}$ of incubation the medium was exchanged for the fresh medium supplemented with chromium chloride or iron chloride at concentrations of 100, 200, 400, 600, 800, 1000, 1200 and $1400 \mu \mathrm{M}$. All concentrations mentioned above are the final concentrations in the incubations. After $24 \mathrm{~h}$ of incubation the MTT reduction assay was performed according to the original manufacturer's instruction by Sigma-Aldrich-In vitro Toxicology Assay Kit MTT based (TOX-1). Results for all experiments represent triplicate determinations.

\section{Detection of anti-HSV-1 activity}

The HEp-2 cells were cultured as adherent monolayers in EMEM supplemented with $10 \%$ (v/v) FBS and Antibiotic/antimycotic solution $(1 \mathrm{ml}$ per $100 \mathrm{ml}$ of cell culture medium). After $24 \mathrm{~h}$ incubation the medium was exchanged respectively for fresh medium (control) or medium supplemented with chromium chloride or iron chloride at concentrations of 200, 400, 600,800 and $1000 \mu \mathrm{M}$ and for simultaneous treatment with chromium chloride at concentrations of 200 , 1000,400 and $800 \mu \mathrm{M}$ and iron chloride at concentrations of $1000,200,800,400 \mu \mathrm{M}$ respectively. Two hundred microliters of virus suspension was added at rate of $10 \mathrm{TCID}_{50} / 0.2 \mathrm{ml}$ on Hep-2 to all treatments. After 48-hour of incubation DNA isolation was performed according to the original manufacturer's instruction by the PathogenFree DNA Isolation Kit (GeneProof) and stored at $-20{ }^{\circ} \mathrm{C}$ until needed. Viral nucleic acid was isolated from $200 \mu$ tissue culture supernatant. The thus obtained DNA was analyzed by Real Time PCR Herpes Simplex Virus (HSV1/2) PCR Kit for 45 cycles with Rotor Gene Q (Corbett Life Science)-Amplification program: UDG decontamination $37{ }^{\circ} \mathrm{C}\left(2 \mathrm{~min}\right.$.), initial denaturation $95^{\circ} \mathrm{C}$ (10 min.), denaturation $95^{\circ} \mathrm{C}(5 \mathrm{~s})$, annealing $60{ }^{\circ} \mathrm{C}$ (40 s) - reading for fluorescence signal, extension $72{ }^{\circ} \mathrm{C}(20 \mathrm{~s})$. Results for all experiments represent triplicate determinations.

Real Time fluorescent measurements were obtained and the threshold cycle $\left(\mathrm{C}_{t}\right)$ value for each sample was calculated by determining the point at which fluorescence exceeded a threshold limit. A standard graph of the $C_{t}$ values obtained in series diluted external DNA standard was prepared. $C_{t}$ values obtained from the samples were plotted on the standard curve, and the number of copies was calculated automatically by the Rotor-Gene Q Series Software 2.0.2 (Build 4).

\section{Detection of anti-BVDV activity}

The BT cells were cultured as adherent monolayers in DMEM supplemented with $10 \%(\mathrm{v} / \mathrm{v}) \mathrm{HS}$ and Antibiotic/antimycotic solution $(1 \mathrm{ml}$ per $100 \mathrm{ml}$ of cell culture medium). After a $24 \mathrm{~h}$ incubation the medium was exchanged respectively for fresh medium (control), or medium supplemented with chromium chloride or iron chloride at concentrations of 200, 400, 600, 800 and $1000 \mu \mathrm{M}$ and for simultaneous treatment with chromium(III) at concentrations of 200, 1000, 400 and 800 and iron(III) at concentrations of 1000, 200, 800, 400 respectively. Two hundred microliters of virus suspension was added at the rate of $10 \mathrm{TCID}_{50} / 0.2 \mathrm{ml}$ on BT cells to all treatments. After 5 days of incubation RNA isolation was performed according to the original manufacture's instruction and stored at $-20^{\circ} \mathrm{C}$ until needed. Viral nucleic acid was isolated from $200 \mu \mathrm{l}$ tissue culture supernatant. Isolation was performed according to the original manufacturer's instruction by the PathogenFree RNA Isolation Kit (GeneProof). The thus obtained RNA was analyzed by Real Time PCR. The test was performed according to the original manufacturer's instruction by the Quantification of Bovine Viral Diarrhoea Virus Kit (Primer Desing). 
One step qRT-PCR combines the reverse transcription and real-time PCR reaction in a simple closed tube protocol. Amplification program: reverse transcription-10 $\mathrm{min}, 55^{\circ} \mathrm{C}$, enzyme activation $-2 \mathrm{~min}$, $95{ }^{\circ} \mathrm{C}$, denaturation $-10 \mathrm{~s}, 95^{\circ} \mathrm{C}-50$ cycles, data collection: $60 \mathrm{secs}, 60{ }^{\circ} \mathrm{C}-50$ cycles. Fluorogenic data was collected through the FAM and VIC channels. Results for all experiments represent triplicate determinations.

RT fluorescent measurements were obtained and the threshold cycle $\left(C_{t}\right)$ value for each sample was calculated by determining the point at which fluorescence exceeded a threshold. A standard graph of $\mathrm{C}_{\mathrm{t}}$ values obtained was plotted on the standard curve, and the number of copies was calculated automatically by the Rotor-Gene Q Series Software 2.0.2 (Build 4).

Statistical analysis of the data

The results were expressed as mean $\pm \mathrm{SD}$ and the data were analyzed by the use of one way analysis of variance (ANOVA) with Tukey's multi-comparison post-test using Statistica programme. In all the cases, $\mathrm{p}<0.05$ was considered significant.

\section{Results}

Cytotoxicity of chromium chloride and iron chloride on HEp-2 and BT cells

In order to determine the efficacy of chromium(III) and iron(III) against HSV1 in vitro, we first analyzed its cytotoxicity on cultured HEp- 2 cells. While, to

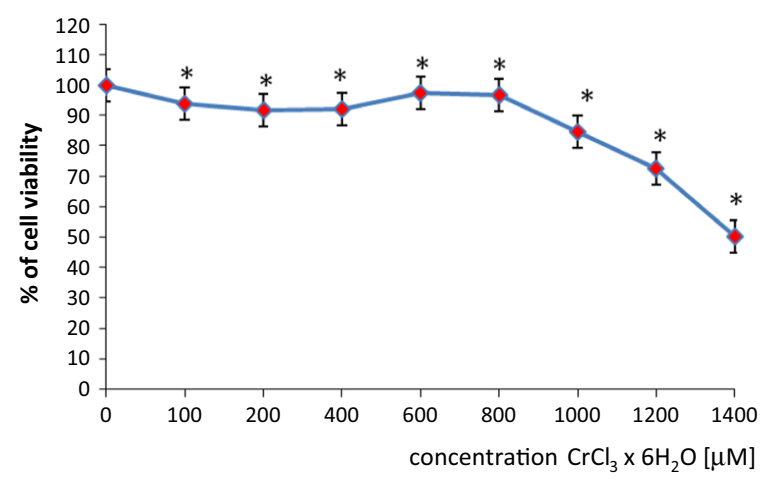

determine the efficacy of chromium(III) and iron(III) against BVDV in vitro, we first analyzed its cytotoxicity on cultured BT cells. Cytotixicity assays are fundamental for the initial phases of antiviral substances development because they define the concentrations to be used. The assesment of the cytotoxicity of new substances is usually performed by cell viability. The MTT assay is probably one of the most widely used assay (Abdel-Rahman et al. 2016). In our study the cytotoxicity of the tested microelements was evaluated by this assay and the $\mathrm{IC}_{50}$ values are expressed in $\mu \mathrm{M}$. The cytotoxicity results of chromiu$\mathrm{m}$ (III) and iron(III) are presented in Figs. 1 and 2. The MTT assay demonstrated a low toxicity of microelements used on HEp-2 cells and BT cells. The $\mathrm{IC}_{50}$ for chromium chloride was $1100 \mu \mathrm{M}$ for Hep-2 cells and $1400 \mu \mathrm{M}$ for BT cells. The $\mathrm{IC}_{50}$ for iron chloride was $1200 \mu \mathrm{M}$ for Hep- 2 cells and more than $1400 \mu \mathrm{M}$ for BT cells. These results are presented in Table 1.

Antiviral activity of chromium chloride and iron chloride

All samples were observed under microscope. When cythopathic effect was observed, RNA was extracted from supernatant of infected BT cells (Fot. 1 and 2) and DNA was extracted from supernatant of infected HEp-2 cells (Fot. 3, 4, 5).

The data of the antiviral activity of chromium chloride and iron chloride used separately are demonstrated in Figs. 3 and 4. Chromium chloride and iron chloride show antiviral effect in all used concentrations which did not affect the cell viability. Figure 3 shows the in vitro effects of iron(III) and

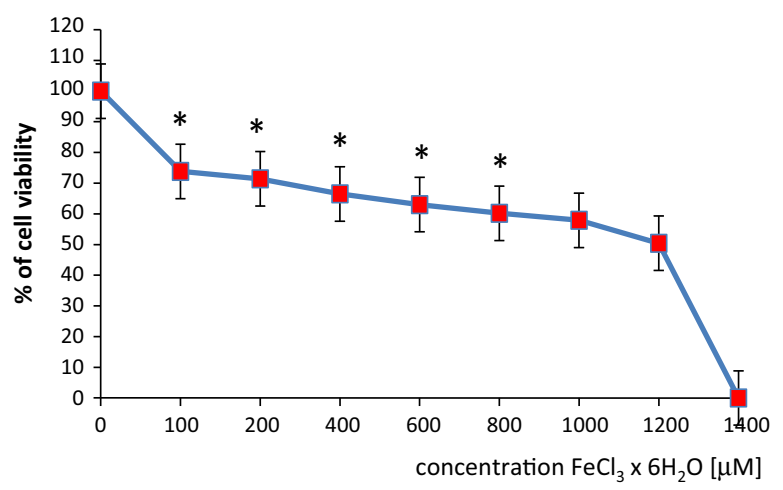

Fig. 1 Cytotoxic effect of chromium chloride or iron chloride in HEp-2 cell line, detected with the MTT reduction assay. Values are given as percentage of cell viability. ${ }^{*} \mathrm{p}<0.05$, significance of difference compared with control 

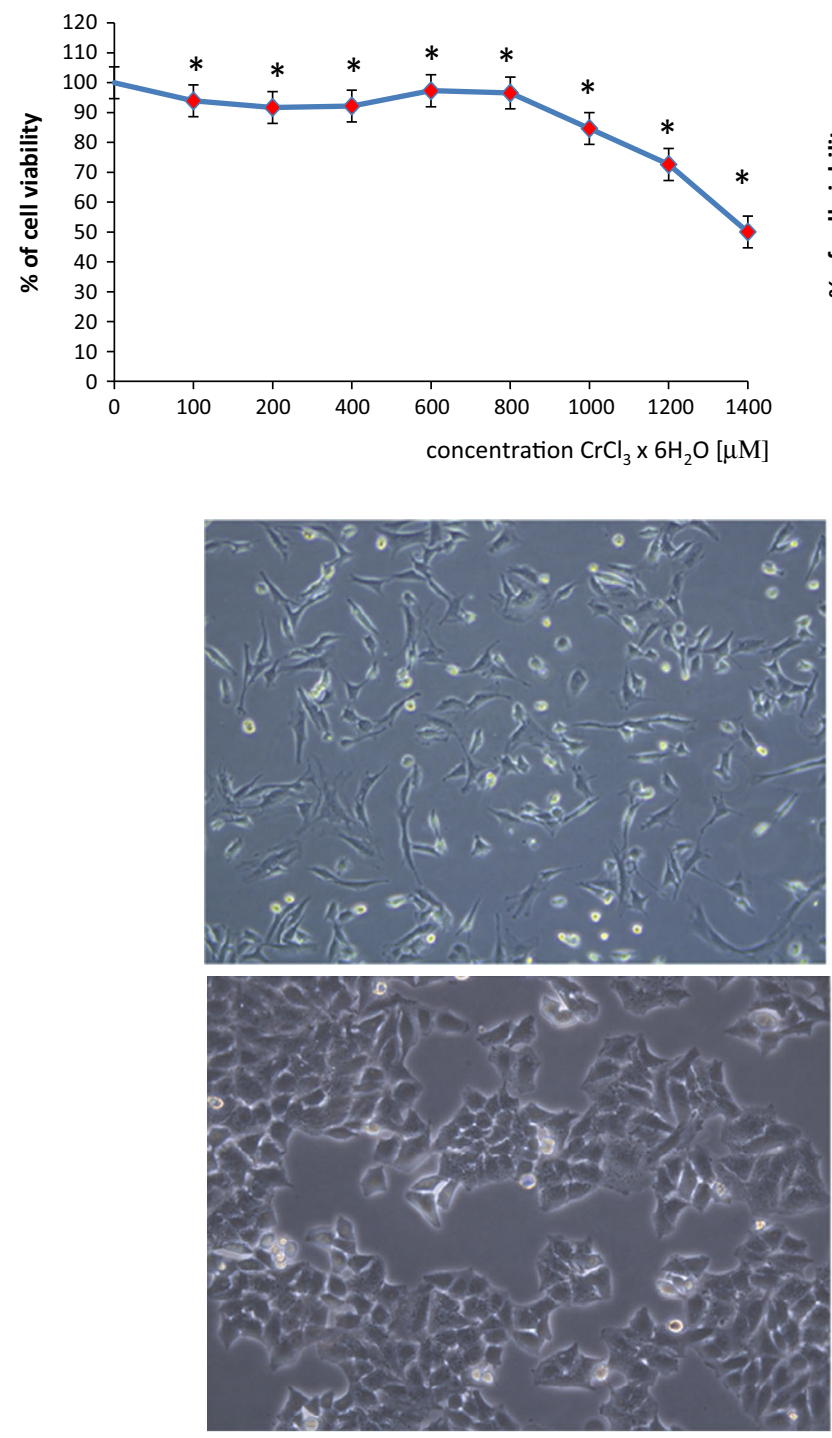

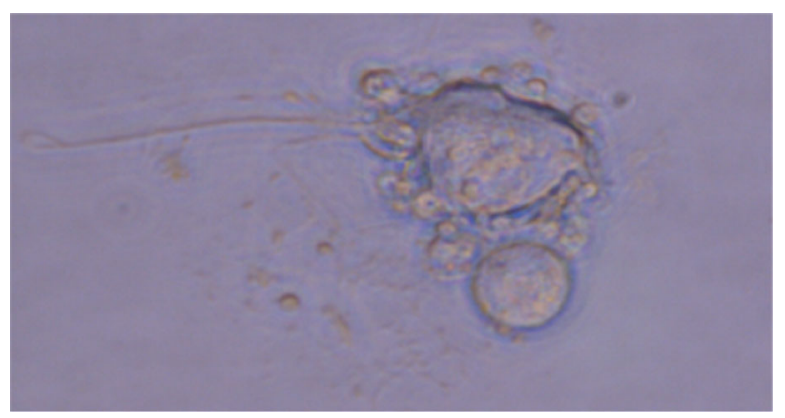

Fig. 2 Cytotoxic effect of chromium chloride or iron chloride in BT cell line detected with the MTT reduction assay. Values are given as percentage of cell viability. ${ }^{*} p<0.05$, significance of difference compared with control. Fot. 1. BT cells (control cells), magnification of $\times 100$, Fot. 2. Cythopathic effect of
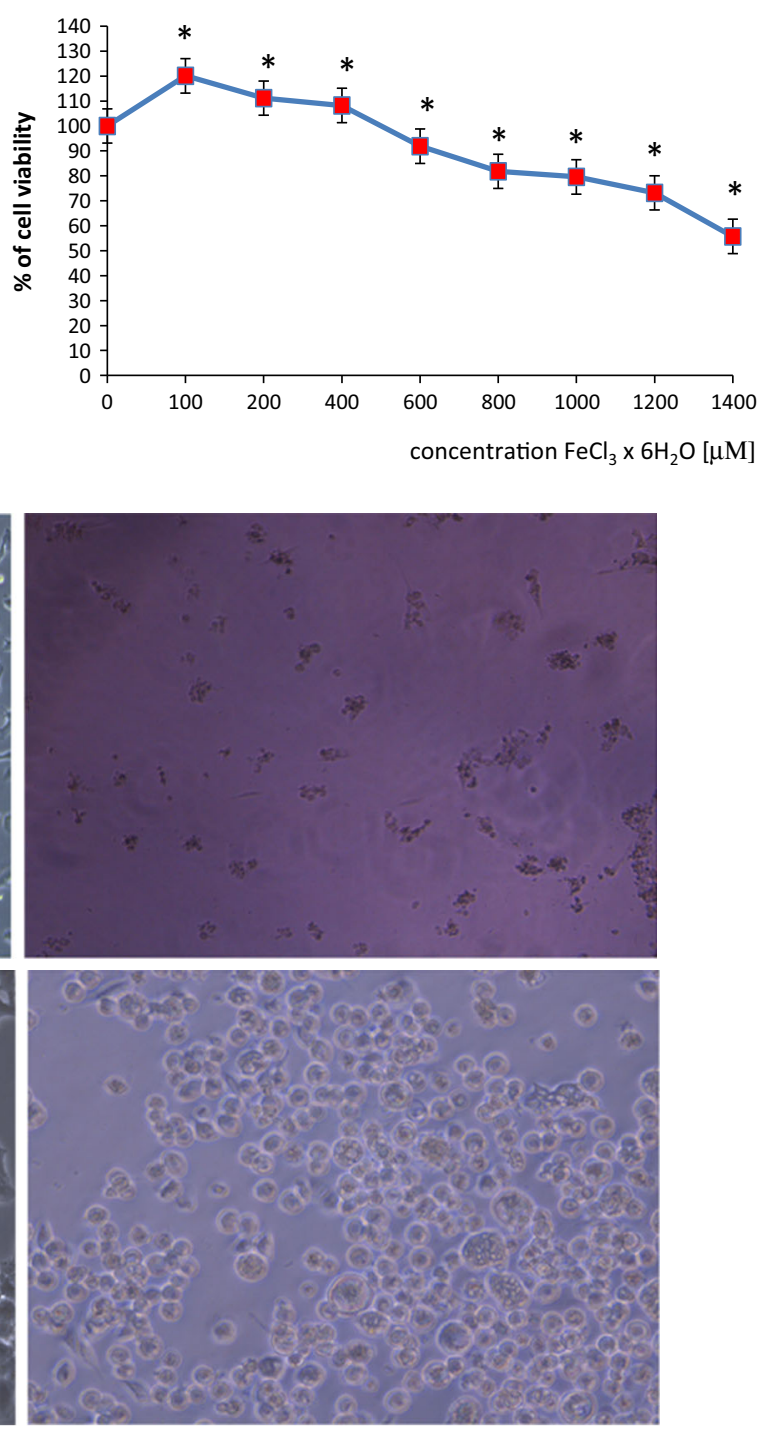

BVDV-1 in BT cells, magnification of $\times 100$, Fot. 3 . HEp- 2 cells (control cells), magnification of $\times 100$, Fot. 4. Cythopathic effect of HSV-1 in HEp-2 cells, magnification of $\times 200$, Fot. 5 . Cythopathic effect of HSV-1 in HEp-2 cells, magnification of $\times 630$ - new viruses released from cells 
Table 1 Cytotoxicity of chromium chloride and iron chloride. Inhibitory concentration $\left(\mathrm{IC}_{50}, \mu \mathrm{M}\right)$

\begin{tabular}{lll}
\hline & HEp-2 line & BT line \\
\hline $\mathrm{CrCl}_{3} \times 6 \mathrm{H}_{2} \mathrm{O}$ & 1100 & 1400 \\
$\mathrm{FeCl}_{3} \times 6 \mathrm{H}_{2} \mathrm{O}$ & 1200 & $>1400$ \\
\hline
\end{tabular}

chromium(III) on number of copies of DNA. Moreover, iron(III) and chromium(III) used separately decrease the number of copies of RNA in all concentrations (Fig. 4).

In cultures simultaneously treated with $200 \mu \mathrm{M}$ of $\mathrm{CrCl}_{3}$ and $1000 \mu \mathrm{M}$ of $\mathrm{FeCl}_{3}, 1000 \mu \mathrm{M}$ of $\mathrm{CrCl}_{3}$ and $200 \mu \mathrm{M}$ of $\mathrm{FeCl}_{3}, 400 \mu \mathrm{M}$ of $\mathrm{CrCl}_{3}$ and $800 \mu \mathrm{M}$ of $\mathrm{FeCl}_{3}, 800 \mu \mathrm{M}$ of $\mathrm{CrCl}_{3}$ and $400 \mu \mathrm{M}$ of $\mathrm{FeCl}_{3}$ a decrease in number of copies of DNA was observed compared with control cells and cells incubated with chromium(III) at concentrations 200 and $400 \mu \mathrm{M}$ and iron(III) used separately (Figs. 5, 6).

In cultures simultaneously treated with $200 \mu \mathrm{M}$ $\mathrm{CrCl}_{3}$ and $1000 \mu \mathrm{M} \mathrm{FeCl}_{3}, 1000 \mu \mathrm{M} \mathrm{CrCl}_{3}$ and $200 \mu \mathrm{M} \mathrm{FeCl}{ }_{3}, 400 \mu \mathrm{M} \mathrm{CrCl}_{3}$ and $800 \mu \mathrm{M} \mathrm{FeCl}$, $800 \mu \mathrm{M} \mathrm{CrCl} \mathrm{Cr}_{3}$ and $400 \mu \mathrm{M} \mathrm{FeCl}{ }_{3}$ a decrease in number of copies of RNA was observed compared with control cells and cells incubated with chromiu$\mathrm{m}$ (III) and iron(III) used separately (Figs. 7, 8).

\section{Disscusion}

The relationship between microelements in in vitro and in vivo experiments has attracted attention of many investigators. Nutrient-nutrient interactions may negatively or positively affect the cell viability and replication of bacteria or viruses.

The concentrations of chromium chloride and iron chloride for these studies were chosen on the basis of other reports (Mazzotti et al. 2001, 2002) and our earlier investigations. Our previous experiments have shown that both of them slightly stimulated cell proliferation (Terpiłowska and Siwicki 2009, 2010). Our previous investigations have shown that chromium(III) and iron(III) statistically increase IL- $1 \alpha$ at the concentrations of 50 and $500 \mu \mathrm{M}$, and that they decrease IL-6 concentration when compared with control cells. Simultaneous treatment with chromium and iron suggests the synergistic interaction between these elements (Terpiłowska and Siwicki 2012).

At the molecular level the most promising mechanisms of antiviral intervention may involve: prevention of the formation of the virus protein's active configuration (e.g. by blocking normal homodimer or heterodimer formation) or elimination of enzymatic function by blockage or distortion of the active site through strong or irreversible binding of a specific antimetabolite; or subversion of the protein's normal function towards lethal synthesis-for example by provision of an innocuous substrate for conversion in the virus infected cells into a DNA chain terminator or destabilizer or antimetaboite (Subak-Sharpe and Darga 1998).

The first antivirals, 5-Iodo-2'-deoxyiuridine (IDU) and 5-Trifluoromethyl- $2^{\prime}$-deoxyuridine (TFT), date from the 1960s, and until today, they have been still used. The first antiviral compound used in the systemic treatment of herpesvirus infections was vidarabine. It was then superseded by acyclovir, which appeared to be more selective in its activity against HSV because of a specific phosphorylation by the virus-induced tymidine kinase (TK) (De Clerq 2013). However, a serious problem in the use of acyclovir is drug resistance in patients. Moreover, these drugs are expensive and several patients with frequent attacks may not be able to afford the cost of long-term treatment (Khan et al. 2005). There is a great

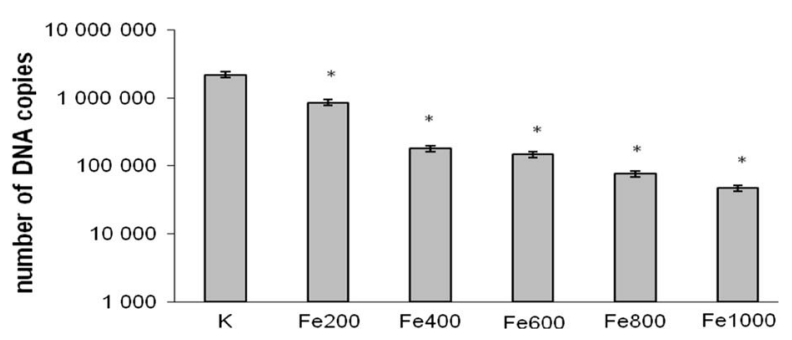

Fig. 3 Effect of chromium chloride or iron chloride on HSV-1 replication. *p $<0.05$, significance of difference compared with control 

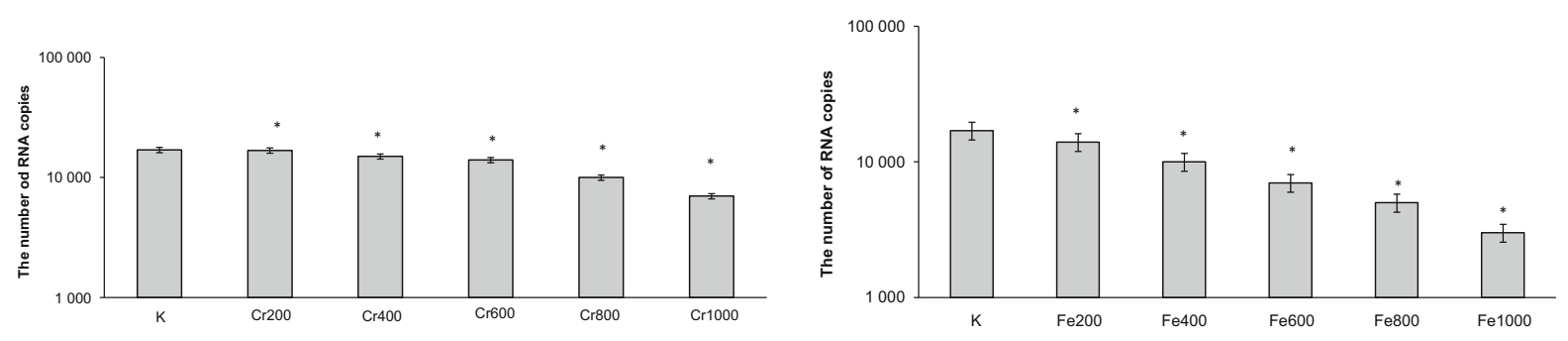

Fig. 4 Effect of chromium chloride or iron chloride on BVDV replication. ${ }^{\mathrm{p}} \mathrm{p}<0.05$, significance of difference compared with control

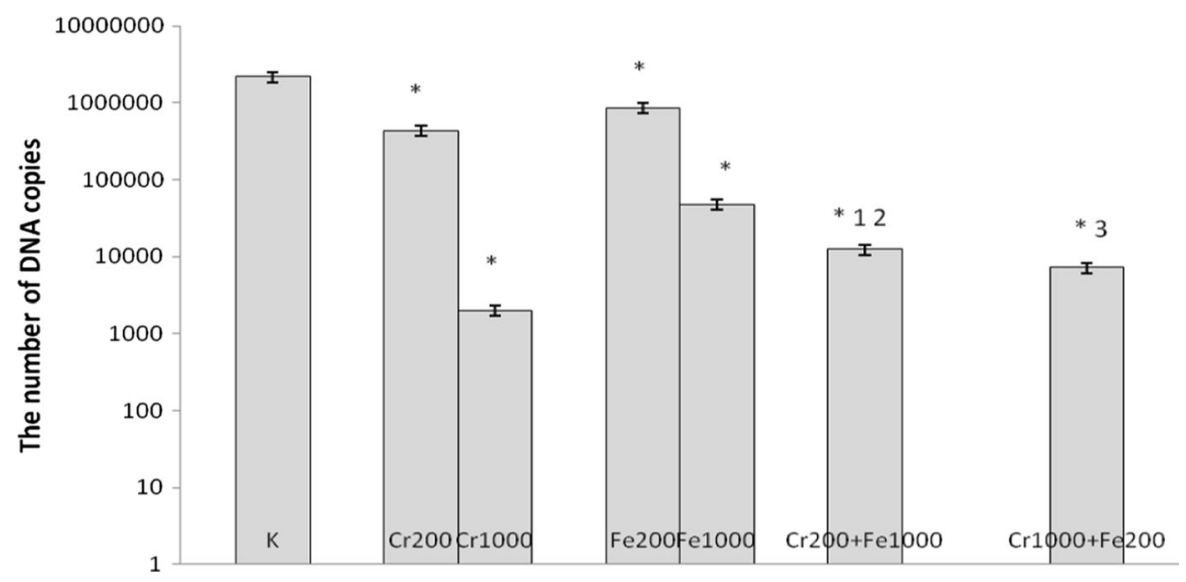

Fig. 5 Effects of chromium chloride and iron chloride on number of copies of DNA. * $\mathrm{p}<0.05$, significance of difference compared with control. $1 \mathrm{p}<0.05$, significance of difference compared with chromium chloride at concentration of $200 \mu \mathrm{M}$;
2 significance of difference compared with iron chloride at concentration of $1000 \mu \mathrm{M} ; 3 \mathrm{p}<0.05$, significance of difference compared with iron chloride at concentration of $200 \mu \mathrm{M}$

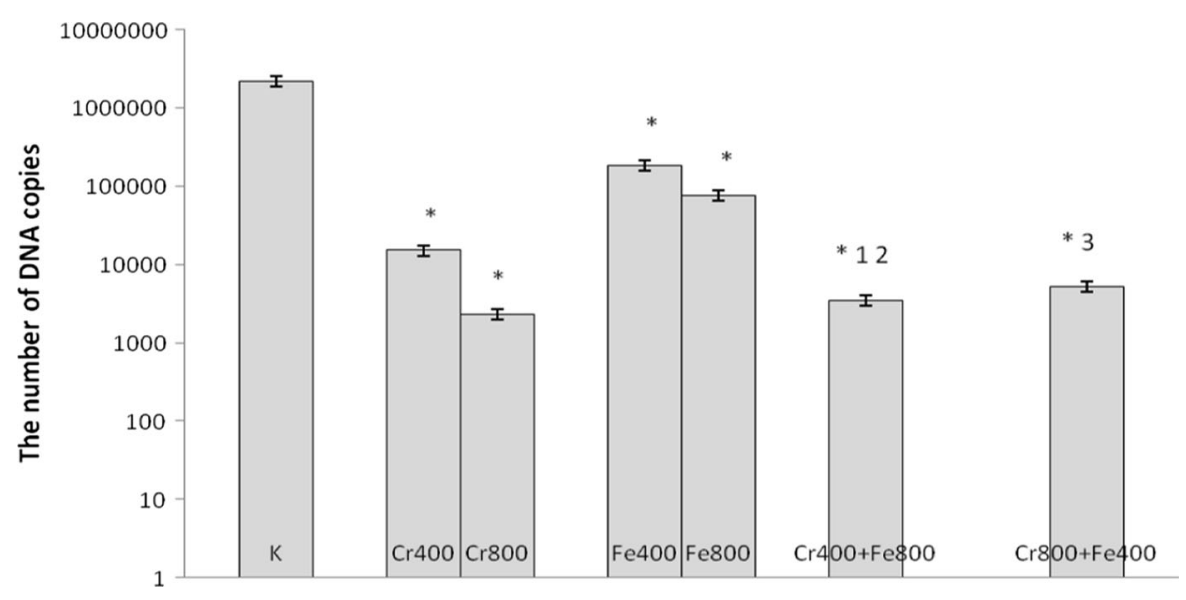

Fig. 6 Effects of chromium chloride and iron chloride on number of copies of DNA. $* p<0.05$, significance of difference compared with control. $1 \mathrm{p}<0.05$, significance of difference compared with chromium chloride at concentration of $400 \mu \mathrm{M}$;

interesting possibility to develop efficacious antiviral compounds being natural products with low toxicity that are well tolerated (Glatthaar-Salmüller et al.

$2 \mathrm{p}<0.05$, significance of difference compared with iron chloride at concentration of $800 \mu \mathrm{M} ; 3 \mathrm{p}<0.05$, significance of difference compared with iron chloride at concentration of $400 \mu \mathrm{M}$

2011). As an alternative, naturally occurring substances found in plants and fruit could be cheaper and more efficacious to use (Danaher et al. 2011). These 


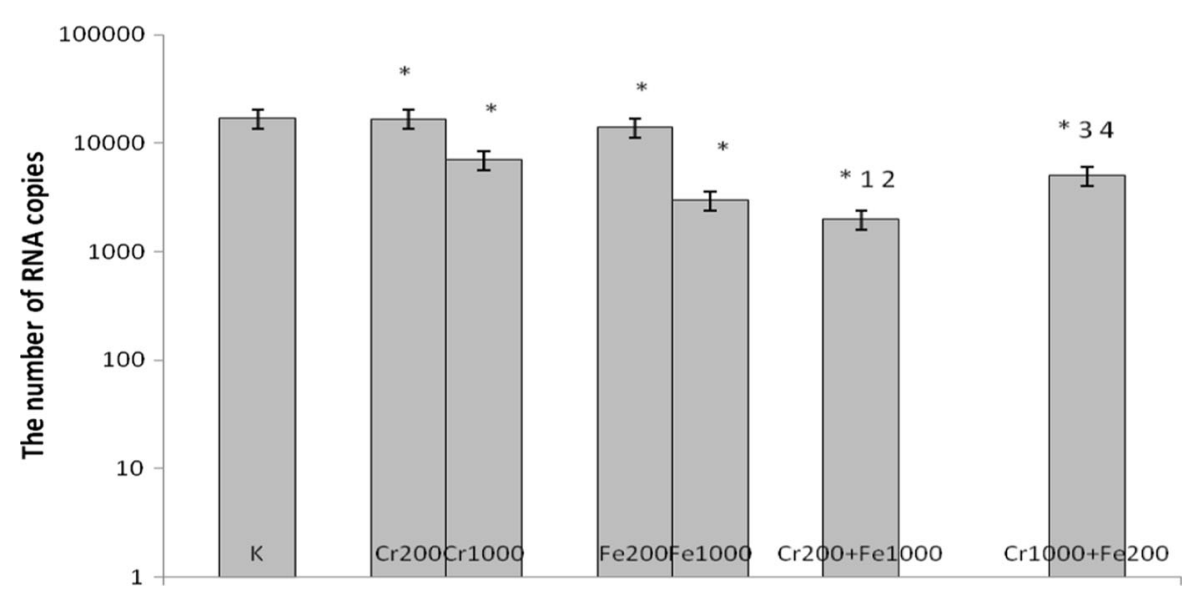

Fig. 7 Effects of chromium chloride and iron chloride on number of copies of RNA. *p $<0.05$, significance of difference compared with control. $1 \mathrm{p}<0.05$, significance of difference compared with chromium chloride at concentration of $200 \mu \mathrm{M}$; $2 \mathrm{p}<0.05$, significance of difference compared with iron chloride at concentration of $1000 \mu \mathrm{M} ; 3 \mathrm{p}<0.05$, significance of difference compared with chromium chloride at concentration of $1000 \mu \mathrm{M} ; 4 \mathrm{p}<0.05$, significance of difference compared with iron chloride at concentration of $200 \mu \mathrm{M}$

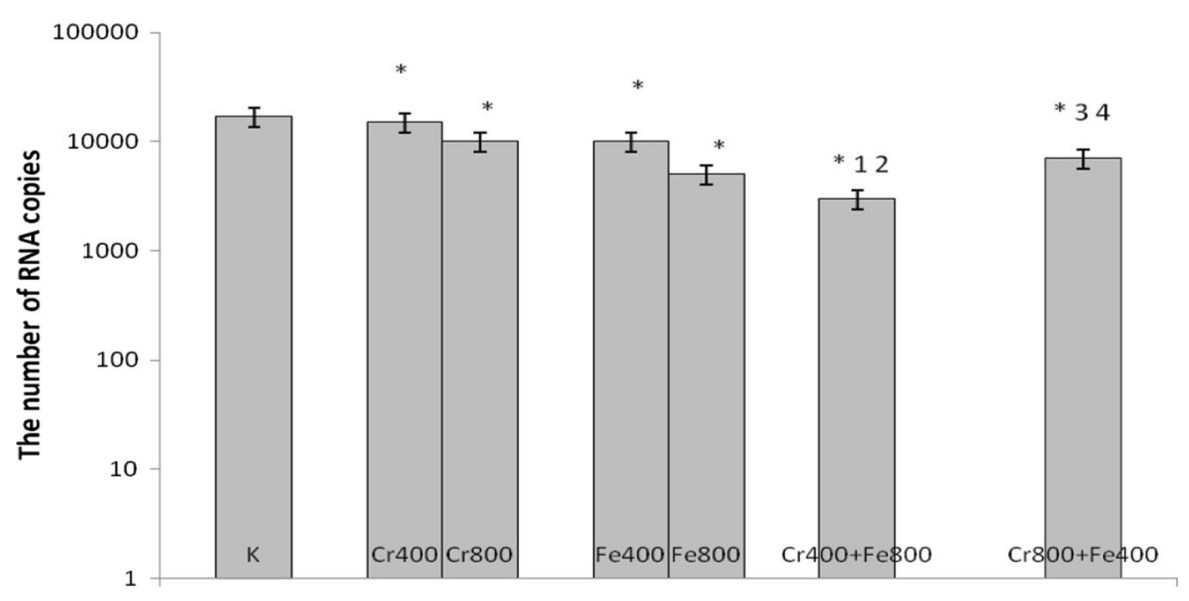

Fig. 8 Effects of chromium chloride and iron chloride on number of copies of RNA. *p $<0.05$, significance of difference compared with control. $1 \mathrm{p}<0.05$, significance of difference compared with chromium chloride at concentration of $400 \mu \mathrm{M}$; $2 \mathrm{p}<0.05$, significance of difference compared with iron

natural substances, which are food component, are easily consumed to prevent viral infections. The extracts from seeds, plants, roots and fruit have been shown to have antiviral activities (Danaher et al. 2011).

There are several in vitro investigations to study the anti-herpetic activities of plant/herbal extracts or plant-derived molecules. The anti-HSV activity of moronic and betulonic acids from the herbal extract of Rhus javanica has been reported. Moreover, the aqueous extracts and pure compounds of Plantago chloride at concentration of $800 \mu \mathrm{M} .3 \mathrm{p}<0.05$, significance of difference compared with chromium chloride at concentration of $800 \mu \mathrm{M} ; 4 \mathrm{p}<0.05$, significance of difference compared with iron chloride at concentration of $400 \mu \mathrm{M}$

major $\mathrm{L}$ have been reported as antiviral (on HSV-1, -2 and adenoviruses-3, -8, 11) agent (Khan et al. 2005). Polyphenols derived from plants have been shown to have antiviral activity. Especially, the flavonoids, galangin, quercitin, procyanidin, and pelargonidin are found to be virucidal against HSV (Danaher et al. 2011). Danaher et al. (2011) shows that blackberry extract has strong antiviral- anti HSV effects that interfere with absorption or entry into host cells and some intracellular activity. Glatthaar-Salmüller et al. (2011) reported concentration-dependent antiviral 
activity $\left(\mathrm{EC}_{50}\right.$ between 13.8 and $\left.124.8 \mu \mathrm{g} / \mathrm{ml}\right)$ of Sinupret against RNA and DNA viruses. Sinupret is herbal medicine product made from Gentian root, Primula flower, Sorrel herb and Verbena herb. Moreover, metal nanoparticles were found to be virucidal against DNA and RNA viruses. Metal nanoparticles such as silver and gold have demonstrated efficient inhibitory activity against Human Immunodeficiency Virus, Hepatitis B Virus, influenza virus and Peste des petits ruminants virus (Hang et al. 2015). Hang et al. (2015) demonstrated that cuprous nanoparticle (at cytotoxic concentration) significantly inhibited Hepatitis C virus infection in the HCVcc/Huh7.5.1 cell culture system. Furthermore, it was indicated that cuprous oxide, sulphide, iodide and chloride have highly efficient antiviral activities- on bacteriophage QB (Sunada et al. 2012). Nickel was another tested element. The antiviral activity of Ni-chitosan microcomposite affects the free virus adsorption to the cells by binding to the VP1 protein of enterovirus 71 (Lin and Chang 2014). Investigation conducted by AbdelRahman et al. (2016) shows that nanoCr(III) and nanoFe(II) complexes inhibit HSV and Tobacco Mosaic Viruses (TMV) replication. Minimum inhibitory concentration required to reduce virus-induced cytopathogenicity by $50 \%$ was $126.4 \mu \mathrm{g} / \mathrm{ml}$ for nanoCr(III) and $12.3 \mu \mathrm{g} / \mathrm{ml}$ for nanoFe(II) against HSV. Minimum inhibitory concentration required to reduce virus-induced cytopathogenicity by $50 \%$ was $70 \mu \mathrm{g} / \mathrm{ml}$ for nanoCr(III) and $0,83 \mu \mathrm{g} / \mathrm{ml}$ for nano$\mathrm{Fe}(\mathrm{II})$ against TMV.

Possible mechanisms of the antiviral activity include: interaction with the viral surface, interference with the viral attachment, inhibition of virus penetration into the cell, interaction with the viral genome, inhibition of genome replication, inhibition of protein synthesis and inhibition of assembly and release of virions (Hang et al. 2015). In this study, we demonstrated a decrease in number of copies of DNA of HSV after a $48 \mathrm{~h}$ of incubation or RNA of BVDV after 5 days of incubations. Here we report the antiviral activities of chromium(III) and iron(III) against HSV1 and BVBV. For HSV-1, synergistic effect was found at higher concentrations of chromium chloride, i.e. 800 and $1000 \mu \mathrm{M}$ combined with iron chloride (200, $400,800$ and $1000 \mu \mathrm{M})$. For BVDV1, synergistic effect was found for chromium chloride combined with iron chloride. The mechanism of antiviral synergism between chromium and iron has not been determined. It has been demonstrated that $\mathrm{Cr}$ (III) compounds can bind directly to DNA or RNA in vitro, forming Cr-DNA adducts and DNA-DNA crosslinks (O'Brien et al. 2003). Moreover, Cr(III) has been shown to be able to increase the catalytic activity and decrease the fidelity of DNA polymerase (Galaris and Evangelou 2002). Moreover, excess free iron(III) promotes the formation of reactive oxygen species (ROS), which attack cellular nucleic acids (Andrews 2000, 2005). It is assumed that chromium(III) and iron(III) used separately and simultaneously may interact with viral DNA or RNA and this interaction may result in interaction with the viral genome and blocking the genome replication.

Chromium(III) and iron(III) are essential trace elements in the human body, which play a crucial role in the biochemistry of all living organisms (Hang et al. 2015). Nutrition plays an important role in the development and also in the prevention of cancer, cardiovascular diseases, and diabetes. Chromium(III) and iron(III) are trace elements necessary for growth and normal functioning of cells. We observed significant inhibitory effects of chromium(III) and iron(III) against HSV and BVDV, at non cytotoxic concentrations in the HEp-2 and BT cells. Thus, these problems still demand a lot of investigation. Future research is warranted to assess their potential for practical use in clinical settings.

\section{Compliance with ethical standards}

Conflict of interest The authors declare that they have no conflict of interest.

Open Access This article is distributed under the terms of the Creative Commons Attribution 4.0 International License (http:// creativecommons.org/licenses/by/4.0/), which permits unrestricted use, distribution, and reproduction in any medium, provided you give appropriate credit to the original author(s) and the source, provide a link to the Creative Commons license, and indicate if changes were made.

\section{References}

Abdel-Rahman LH, Abu-Dief AM, Newair EF, Kamel Hamdan S (2016) Some new nano-sized Cr(III), Fe(II), Co(II), and $\mathrm{Ni}(\mathrm{II})$ complexes incorporating 2-((E)-(pyridine-2-ylimino)methyl)napthalen-1-ol ligand: structural characterization, electrochemical, antioxidant, antimicrobial, antiviral assessment and DNA interaction. J Photochem Photobiol B Biol 160:18-31. doi:10.1016/j.jphotobiol.2016.03.040 
Andrews NC (2000) Intestinal iron absorption: current concepts circa 2000. Diges Liver Dis 32:56-61

Andrews NC (2005) Molecular control of iron metabolism. Best Pract Res Clin Haematol 8(2):159-169

Cermelli C, Vincenti M, Scaltriti E, Bazzani E, Beretti F, Vivoli G, Portolani M (2002) Selenite inhibition of Coxsackie virus B5 replication: implications on the etiology of Keshan disease. J Trace Elem Med Biol 16:41-46. doi:10. 1016/S0946-672X(02)80007-4

Chai H, Lim D, Chai H, Jung E (2013) Molecular modeling of small molecules as BVDV RNA-dependent RNA polymerase allosteric inhibitors. Bull Korean Chem Soc 34(3):837-850. doi:10.5012/bkcs.2013.34.3.837

Danaher RJ, Wang C, Dai J, Mumper RJ, Miller CS (2011) Antiviral effects of blackberry extract against herpes simplex virus type 1. Oral Surg Oral Med Oral Phatol Oral Radiol Endod 112:e31-e35. doi:10.1016/j.tripleo.2011.04. 007

De Clerq E (2013) Antivirals: past, present and future. Biochem Pharmacol 85:727-744. doi:10.1016/j.bcp.2012.12.011

Galaris D, Evangelou A (2002) The role of oxidative stress in mechanisms of metal-induced carcinogenesis. Crit Rev Oncol Hematol 142:93-103

Glatthaar-Salmüller B, Rauchhaus U, Rode S, Haunschild J, Salmüller A (2011) Antiviral activity in vitro of two preparations of the herbal medicinal product Sinupret against viruses causing respiratory infections. Phytomedicine 19:1-7. doi:10.1016/j.phymed.2011.10.010

Haase H, Overbeck S, Rink L (2008) Zinc supplementation for the treatment or prevention of disease: current status and future perspectives. Exp Gerontol 43:394-408. doi:10. 1016/j.exger.2007.12.002

Hang X, Peng H, Song H, Qi Z, Miao X, Xu W (2015) Antiviral activityof cuprous oxide nanoparticles against Hepatitis $\mathrm{C}$ virus in vitro. J Virol Methods 222:150-157. doi:10.1016/j. jviromet.2015.06.010

He DX, Tam SC (2010) Trichosanthin affects HSV-1 replication in Hep-2 cells. Biochem Biophys Res Comm 402:670-675. doi:10.1016/j.bbrc.2010.10.080

Khan MTH, Ather A, Thompson KD, Gambari R (2005) Extracts and molecules from medicinal plants against herpes simplex viruses. Antiviral Res 67:107-119. doi:10. 1016/j.antiviral.2005.05.002

Kim SG, Anderson RR, Yu JZ, Zylich NC, Kinde H, Carman S, Bedenice D, Dubovi EJ (2009) Genotyping and phylogenetic analysis of bovine viral diarrhea virus isolates from BVDV infected alpacas in North America. Vet Microbiol 136:209-216. doi:10.1016/j.vetmic.2008.10. 029

Letellier C, Kerkhofs P (2003) Real-time PCR for simultaneous detection and genotyping of bovine Viral Diarrhea Virus. J Virol Meth 114:21-27. doi:10.1016/j.jviromet.2003.08. 004

Lin Y-Ch, Chang Ch-H (2014) In vitro inhibition of enetrovirus 71 infection with nickel ion/chitosa microcomposite. Virus Res 190:17-24. doi:10.1016/j.virusres.2014.06.012

Mazzotti F, Sabbioni E, Ghiani M, Cocco B, Ceccatelli R, Fortaner S (2001) In vitro assessment of cytotoxicity and carcinogenic potential of chemicals: evaluation of induced by 58 metal compounds in the Balb/3T3 cell line. ATLA 29:601-611

Mazzotti F, Sabbioni E, Ponti J, Ghiani M, Fortaner S, Rossi GL (2002) In vitro setting of dose- effect relationships of 32 metal compounds in the Balb/3T3 cell line, as a basis for predicting their carcinogenic potential. ATLA 30:209-217

O'Brien TJ, Ceryak S, Patierno SR (2003) Complexities of chromium carcinogenesis: role of cellular response, repair and recovery mechanisms. Mutat Res 533:3-36. doi:10. 1016/j.mrfmmm.2003.09.006

Subak-Sharpe JH, Darga DJ (1998) HSV Molecular biology: general aspects of Herpes Simplex Virus molecular biology. Virus Genes 16(3):239-251

Sunada K, Minoshima M, Hashimoto K (2012) Highly efficient antiviral and antibacterial activities of solid-state cuprous compounds. J Hazard Mater 235-236:265-270. doi:10. 1016/j.jhazmat.2012.07.052

Terpiłowska S, Siwicki AK (2009) The influence of iron on cellmediated and humoral-mediated immunity in mice. Cent Eur J Immunol 34(2):57-60

Terpiłowska S, Siwicki AK (2010) The influence of chromium on cell-mediated and humoral-mediated immunity in mice. Cent Eur J Immunol 35(1):10-13

Terpiłowska S, Siwicki AK (2012) The influence of chromium and iron interactions an cell viability. Toxicol Lett 2115:S43-S216. doi:10.1016/j.toxlet.2012.03.384

Zhang S, Tan B, Ding Y, Wang F, Guo L, Wen Y, Cheng S, Wu $\mathrm{H}$ (2014) Complete genome sequence and pathogenesis of bovine diarrhea virus JL-1 isolate from cattle in China. Virol J 11:67. doi:10.1186/1743-422X-11-67 